\title{
Difficulties in the calculation of electron spin resonance parameters using density functional methods
}

\author{
H.U. Suter ${ }^{1}$, V. PleB, M. Ernzerhof, B. Engels * \\ Institut für Physikalische und Theoretische Chemie, Universität Bonn, Wegelerstrasse 12, D.53115 Bonn, Germany
}

Received 22 July 1994; in final form 27 September 1994

\begin{abstract}
Density functional theory is applied to the calculation of the isotropic hyperfine coupling constants in some small molecules. Various functionals are tested. The agreement of the calculated values to experimental data and values obtained from sophisticated $a b$ initio methods depends on the functionals used and the system under consideration. With respect to spin density calculations the functional of Lee, Yang and Parr with Becke's exchange functional (BLYP) is found to give good results for the heavier center of the $\mathrm{CH}$ and the $\mathrm{NH}$ molecule, while the spin densities of other molecules such as $\mathrm{OH}, \mathrm{H}_{2} \mathrm{CN}_{,} \mathrm{H}_{2} \mathrm{CO}^{+}, \mathrm{NO}^{2}$ and $\mathrm{O}_{2}$ deviate considerably from experimental and/or other theoretical results $(30 \%-60 \%)$. In cases where the singly occupied orbital can contribute to the isotropic hyperfine coupling constants, accurate results are obtained. The reason for this is analyzed.
\end{abstract}

\section{Introduction}

The analysis of the magnetic hyperfine interaction is generally undertaken in terms of isotropic and anisotropic hyperfine coupling constants ( $\mathrm{hfccs}$ ). The isotropic hfce is proportional to the spin density at the various nuclei while the anisotropic hfcc represents the dipole-dipole interaction of the electronic and the nuclear spins. The calculation of the isotropic hfcc has led to severe problems for conventional ab initio methods $[1,2]$. These problems have been overcome recently using appropriate $\mathrm{AO}$ basis sets [3] and reasonable correlation treatments, as for example the coupled cluster method [4] including triples correction (CCSD $(T)$ ), or the MRD-CI/ $\mathrm{B}_{\mathrm{K}}$ method in which the wavefunction obtained from an

\footnotetext{
- Corresponding author.

'Present address: Centro Svizzero di Calcolo Scientifico, Via Cantonale, CH-6928 Manno, Switzerland.
}

individually selected MRD-CI treatment is corrected perturbationally $[5,6]$.

Due to the development of improved functionals [7-11] density functional theory (DFT) has gained renewed interest. The advantage of DFT calculations is the lower computational cost compared to ab initio methods including a correlation treatment. The DFT method has also been used to calculate hfccs [12-15]. Using the functional proposed by Perdew and Wang in 1986 (PW86) [9] Eriksson et al. [13] found good agreement with experimental results for various systems. However, depending on the functional, for some systems poor results were obtained. In $\mathrm{H}_{2} \mathrm{O}^{+}$the spin density at the hydrogen center is described fairly well by various functionals, whereby the best agreement to the experimental results is obtained if an improved functional of Perdew and Wang (PW91) [10] is used (DFT $=-24.6 \mathrm{G}$, exp. $=-26.1 \mathrm{G}$ ). The spin density at the oxygen center, however, is poorly calculated. For example, a value of $0.6 \mathrm{G}(\exp .=-29.7$ G) is obtained if the PW91 functional is employed. 
Fairly good agreement $(-24.6 \mathrm{G})$ is obtained if the older PW86 functional is used. The performance of various functionals and the success of gradient-corrected functionals was studied by Ishii and Shimizu [14] and Eriksson et al. [12,13]. Gradient-corrected functionals improve the spin densities with respect to the LDA approximation. As shown by Ziegler et al. [16] gradient corrections remove density from the tails of the valence regions and enhance the core density.

However, an interesting effect found for the $\mathrm{H}_{2} \mathrm{CO}^{+}$ molecule was not discussed. Using DFT (PW86 functional) $A_{\text {iso }}\left({ }^{\prime} \mathrm{H}\right)$ is calculated to be $117 \mathrm{G}$ $\left(R_{\mathrm{CH}}=110 \mathrm{pm}, R_{\mathrm{CO}}=121 \mathrm{pm}, \angle \mathrm{HCH}=124^{\circ}\right)$ which is similar to the value of $115 \mathrm{G}$ obtained with the MRD-CI/ $\mathrm{B}_{\mathrm{K}}$ treatment employing a very similar geometry $\left(R_{\mathrm{CH}}=111 \mathrm{pm}, R_{\mathrm{CO}}=121 \mathrm{pm}, \angle \mathrm{HCH}=\right.$ $\left.122^{\circ}\right)$. The excellent agreement is astonishing because as described elsewhere [6] $A_{\text {iso }}\left({ }^{\prime} \mathrm{H}\right)$ strongly depends on correlation effects and within ab initio treatments highly correlated wavefunctions are necessary to obtain such a large value. Surprisingly, the deviation between DFT and MRD-Cl/ $\mathrm{B}_{\mathrm{K}}$ is larger for the heavier centers (carbon, oxygen) which are much easier to describe in ab initio treatments $\left(A_{\text {iso }}\left({ }^{13} \mathrm{C}\right)\right.$ : $\mathrm{DFT}=28.1 \mathrm{G}, \mathrm{MRD}-\mathrm{Cl} / \mathrm{B}_{\mathrm{K}}=37.1 \mathrm{G}$, exp. $38.9 \mathrm{G}$ ).

The aim of this study is to obtain an understanding of the effect by comparing DFT results to ab initio data obtained with accurate treatments such as MRD$\mathrm{Cl} / \mathrm{B}_{\mathrm{K}}[5]$ or $\operatorname{CCSD}(\mathrm{T})$. If $\mathrm{DFT}$ results are compared to accurate $a b$ initio data effects arising from the nuclear motion can be disregarded and furthermore the different contributions to the spin densities can be compared. Calculations were performed for $\mathrm{H}_{2} \mathrm{CN}$ and $\mathrm{H}_{2} \mathrm{CO}^{+}$where the spin densities at the heavier centers are solely due to spin polarization effects. Besides spin polarization effects direct contributions arising from the singly occupied orbital (SOMO) also influence $A_{\text {iso }}\left({ }^{1} \mathrm{H}\right.$ ). Other test systems are typical $\pi$ radicals $\left(\mathrm{CH}, \mathrm{NH}, \mathrm{OH}, \mathrm{NO}, \mathrm{O}_{2}\right.$ ) where the spin densities at both centers result from spin polarization effects only.

\section{Methods of computations}

The calculations were performed with the G92/ DFT program [17]. Three types of functionals have been chosen. For the local density approximation (LDA) we used the Vosko-Wilk-Nusair (VWN) parametrization [18] of the Ceperly and Alder results [19] of the homogeneous electron gas. The Becke gradient corrected exchange functional (BVWN) [7] was used. In addition the functional of Lee, Yang and Parr [11] with Becke's exchange functional (BLYP) and the Becke-Perdew functional (BP86) [8] was employed.

The Gaussian AO basis set was the van Duijneveldt basis as described in Ref. [6] for the $H, C, N$ and $O$ atoms. For the heavier elements it consisted of a $(13 s, 9 p)$ AO basis set proposed by van Duijneveldt [20], contracted to $(8 s, 5 p)$ and augmented with $\mathrm{d}$ polarization functions with exponents $0.318,1.097$ for carbon, $0.645,2.314$ for oxygen and $0.469,1.645$ for nitrogen. The hydrogen is described by a (9s) primitive set contracted to [7s] and augmented by two $\mathrm{p}$ polarization functions with exponents 0.388 , 1.407. In MRD-Cl/ $B_{K}$ calculations this $A O$ basis set was found to be nearly saturated with respect to the isotropic hyperfine coupling constants [6]. Basis sets optimized within the Hartree-Fock scheme could give poorer results in DFT computations. However, the basis sets used in the present work are quite large and only minimally contracted. Therefore we expect only small effects due to basis set incompleteness.

To compare with results given by Eriksson et al. [13] computations were also carried out with the IGLO-III basis set proposed by Kutzelnigg and coworkers [21]. This set is a [7s6p] contraction of the (11s7p) basis of Huzinaga [22] augmented by two d polarization functions. The van Duijneveldt and the IGLO-III basis set should be of similar quality.

\section{Results and discussion}

For the following discussion it is helpful to define 'direct' and 'indirect' contributions. In treatments which employ a one-particle basis to describe the electronic structure, i.e. all ab initio treatments and the Kohn-Sham approach to DFT [23] employed in the present work, two different contributions to the spin density at a given center can be distinguished. The first part is proportional to the spin density of the singly occupied orbital (SOMO) at the center under consideration. In the following it will be called 
the 'direct contribution'. The difference between the direct contribution and the total spin density at the given center is summarized as the indirect contributions'. They arise since the interaction of an unpaired electron with the $\alpha$ and $\beta$ electron of an electron pair is different, leading to small differences in the spatial density distribution of both electrons and thus to a net spin density at all centers. These effects are also called 'spin polarization effects'.

The results obtained for some hydrides of the first row are given in Table 1 . In all molecules the spin density at both centers is solely determined by spin polarization effects because the singly occupied orbitals (SOMO) possess $\pi$ symmetry. Difference between the van Duijneveldt AO basis and the IGLOIII basis are small. The isotropic hfecs at the hydrogen centers depend only slightly on the employed functionals. The improvement with respect to UHF

Table 1

Isotropic hfecs of some hydrides in $\mathrm{G}$

\begin{tabular}{|c|c|c|c|c|}
\hline & Functional & Basis & $\mathrm{X}$ & $\mathrm{H}$ \\
\hline $\mathrm{CH}$ & UHF & Duj & 36 & $-3 t$ \\
\hline \multirow[t]{5}{*}{$x^{2} \Pi$} & BVWN & Duj & 16 & -15 \\
\hline & BP86 & Duj & 9 & -16 \\
\hline & BLYP & Duj & 19 & -15 \\
\hline & MRD-Cl [5] & Duj & 16 & -21 \\
\hline & $\exp .[24]$ & & $17 \pm 1$ & $-21 \pm 0.3$ \\
\hline \multirow{6}{*}{$\begin{array}{l}\mathrm{NH} \\
\mathrm{X}^{3} \Sigma^{-}\end{array}$} & UHF & Duj & 14 & -35 \\
\hline & BVWN & Duj & 6 & -17 \\
\hline & BP86 & Duj & 3 & -18 \\
\hline & BLYP & Duj & 6 & -18 \\
\hline & MRD-CI [25] & Duj & 6 & -23 \\
\hline & $\exp \cdot[26]$ & & 7 & -23 \\
\hline $\mathrm{OH}$ & UHF & Duj & -34 & -39 \\
\hline \multirow[t]{11}{*}{$\mathrm{X}^{2} \Pi$} & BVWN & Duj & -11 & -20 \\
\hline & BP86 & Duj & -6 & -21 \\
\hline & BLYP & Duj & -12 & -22 \\
\hline & UHF & IGLO-III & -34 & -37 \\
\hline & BVWN & 1GLO-11I & -11 & -19 \\
\hline & BP86 & IGLO-III & -7 & -19 \\
\hline & BLYP & IGLO-111 & -12 & -20 \\
\hline & PW86 [13] & IGLO-III & -18 & -21 \\
\hline & MCSCF [3] & & -15 & -24 \\
\hline & $\mathrm{UCCD}(\mathrm{ST})[4]$ & & -18 & -25 \\
\hline & exp. [27] & & -18 & -26 \\
\hline
\end{tabular}

All calculations were performed at the experimental equilibrium geometries. is large but the computed values for $A_{\text {iso }}\left({ }^{\prime} \mathrm{H}\right)$ are uniformly too high by about $5-6 \mathrm{G}(20 \%-25 \%)$.

As already found by Eriksson et al. [13] the isotropic hfccs of the heavier centers depend strongly on the functionals. In our calculations both the BVWN and BLYP functionals give excellent results for $\mathrm{CH}$ and $\mathrm{NH}$ but are less accurate for the oxygen center in $\mathrm{OH}$. The BP86 gives poor agreement in all cases. The $\mathrm{OH}$ radical was studied by Erikson et al. [13] employing the PW86 functional in combination with the IGLO-III basis. They obtained accurate results for the oxygen center. Since the PW86 functional is not contained in the G92/DFT program package a calculation for the other two hydrides was not possible. Comparing the DFT results to those obtained from sophisticated ab initio treatments excellent agreement exists for the heavier centers if appropriate functionals (BLYP, PW86) are used. However, the hydrogen value which is less dependent on the functional is uniformly too small.

In their study Eriksson et al. showed that the PW86 functional is able to predict accurate spin densities for various systems. One of their examples is $\mathrm{H}_{2} \mathrm{CO}^{+}$. A comparison with our calculations is given in the upper part of Table 2. Because no experimental results exist for the oxygen center we will compare to recent MRD-CI/ $\mathrm{B}_{\mathrm{K}}$ calculations which predict accurate isotropic hfccs of the heavier centers (see Tables $1-4)$ and are much more reliable than SD-CI (single and double excitation $\mathrm{CI}$ ) results. Because $A_{\text {iso }}\left({ }^{1} \mathrm{H}\right)$ strongly depends on the geometry, the geometries of the various calculations are given in the lower part of Table 2. For a better comparison of the various functionals we performed most of our calculations with the geometry obtained with a $6-31 \mathrm{G}^{*} / \mathrm{QCISD}(\mathrm{T})$ calculation. This geometry is similar to that given by Feller and Davidson [1] so that one value given by Eriksson et al. [13] can be added to the comparison.

In the $\mathrm{H}_{2} \mathrm{CO}^{+}$molecule the SOMO represents a $\pi$ in plane orbital located mainly at the oxygen center [6]. Due to the symmetry of the SOMO, the isotropic hfccs of the heavier centers consists only of spin polarization effects (indirect contributions) while for the hydrogen centers both direct and indirect contributions to $A_{\text {iso }}$ exist.

As already found for the hydrides no significant difference exists between the results obtained with the van Duijneveldt AO-basis set and the IGLO-III ba- 
Table 2

Upper part: Isotropic hfces of $\mathrm{H}_{2} \mathrm{CO}^{+}\left({ }^{2} \mathrm{~B}_{2}\right)$ in G. Lower part:

Geometries used for the calculation of the hfces of $\mathrm{H}_{2} \mathrm{CO}^{+}\left({ }^{2} \mathrm{~B}_{2}\right)$ (distances are given in $\AA$, angles in deg)

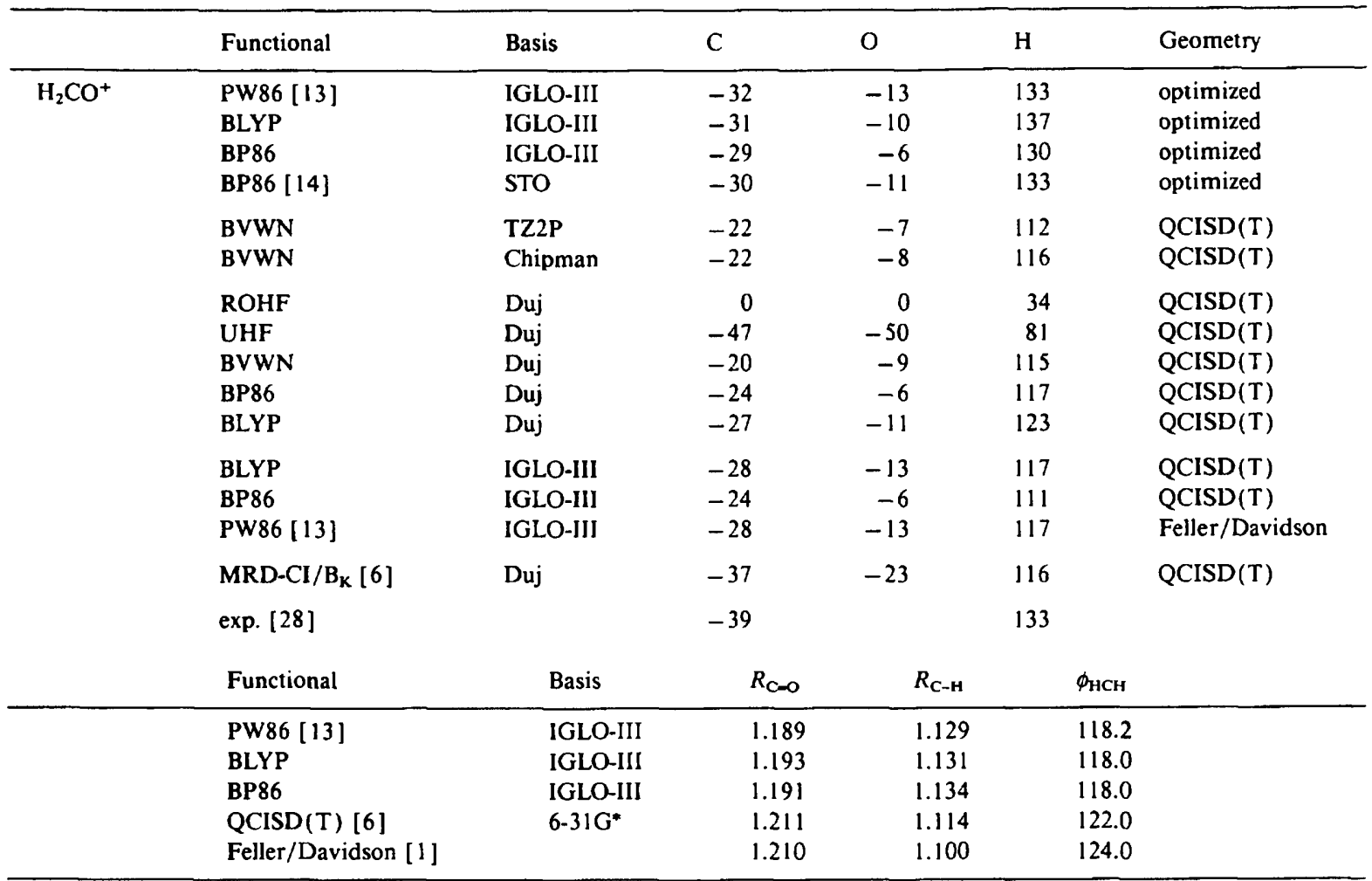

Table 3

Isotropic hfecs of $\mathrm{H}_{2} \mathrm{CN}\left({ }^{2} \mathrm{~B}_{2}\right)$

\begin{tabular}{llrrrr}
\hline Functional & Basis & C & N & H & $H^{\text {a }}$ \\
\hline ROHF & Duj & 0 & 0 & 31 & 31 \\
UHF & Duj & -69 & 26 & 102 & 83 \\
BVWN & Duj & -17 & 6 & 79 & 63 \\
BLYP & Duj & -21 & 7 & 84 & 63 \\
BP86 & Duj & -19 & 4 & 80 & 63 \\
MRD-Cl & Duj [6] & -28 & 9 & 74 & 41 \\
exp. [29] & & -29 & 9 & 83 &
\end{tabular}

The geometry used for these calculations is the MCSCF-ACPF/ Duj geometry $\left(R_{\mathrm{CN}}=1.256 \AA, R_{\mathrm{CH}}=1.094 \AA, \phi_{\mathrm{HCH}}=121.1^{\circ}\right)$ given in Ref. [6]. All values are in $G$.

- Direct contribution, see text for explanation.

sis. Similar to the hydrides the isotropic hfecs of the hydrogen centers depend only slightly on the various functionals $( \pm 3 \%)$. The calculated values are of similar quality as those obtained in the MRD-Cl/ $\mathrm{B}_{\mathrm{K}}$ calculation. Both the BLYP functional used in the present work and the PW86 functional used by Eriksson et al. [13] give $A_{\text {iso }}\left({ }^{\prime} \mathrm{H}\right.$ ) values which deviate less from the experimental data than the MRD-CI/ $\mathrm{B}_{\mathrm{K}} \mathrm{re}$ sults. The agreement with experimental data is even improved if the geometry is optimized using the DFT method itself (Table 2, upper part). However, as already discussed by Eriksson et al. [13] often these geometries are not as good as ab initio data.

For the heavier centers a completely different situation is found. The isotropic hfccs at both centers depend heavily on the functional $( \pm 20 \%)$ and, furthermore, in comparison to the $\mathrm{MRD}-\mathrm{Cl} / \mathrm{B}_{\mathrm{K}}$ treatment the absolute values are much too low. Again BLYP and PW86 (taken from the work of Eriksson et al. [13]) performed best but even for these functionals an error of about $25 \%$ was found for the carbon center while the deviation for the oxygen center was even larger (50\%). While the PW86 functional 
Table 4

Isotropic hyperfine coupling constants of $\mathrm{NO}$ and $\mathrm{O}_{2}$ in $\mathrm{G}$

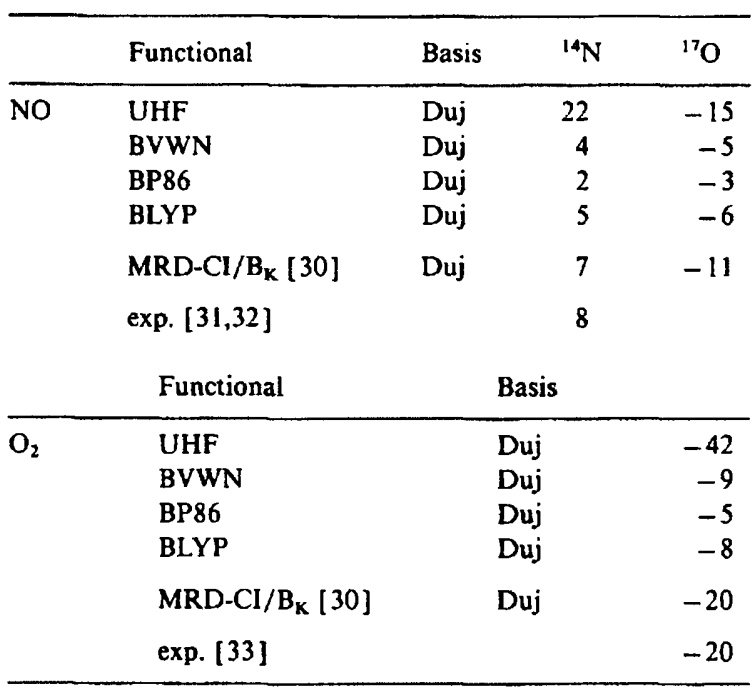

All calculations were performed at the experimental equilibrium geometries $\left(R_{\mathrm{NO}}=2.1751 \mathrm{bohr}, R_{\mathrm{O}_{2}}=2.28 \mathrm{bohr}\right.$ ).

gave excellent results for the oxygen center in $\mathrm{OH}$, it failed for the $\mathrm{H}_{2} \mathrm{CO}^{+}$molecule. To ensure that $\mathrm{H}_{2} \mathrm{CO}^{+}$was not an exception we also performed calculations for $\mathrm{H}_{2} \mathrm{CN}$ (Table 3 ). The results show similar trends as found for $\mathrm{H}_{2} \mathrm{CO}^{+}$. For $\mathrm{H}_{2} \mathrm{CN}$ the BLYP functional which performs excellently in the case of $\mathrm{CH}$ and $\mathrm{NH}$ possesses errors of more than $20 \%$ for the isotropic hfccs of the heavier centers.

Because direct contributions to the hfccs of the heavier centers vanish in both molecules the results indicate that the functionals employed in the comparison are not able to describe spin polarization effects accurately enough. On the other hand, for $A_{\text {iso }}\left({ }^{\prime} \mathrm{H}\right)$, which in the MRD-CI/B $\mathrm{B}_{\mathrm{K}}$ treatment $[6,34]$ is also largely affected by spin-polarization effects, DFT provides excellent values. To get a better insight into this paradoxical situation, we tried to distinguish between the direct and indirect contributions to $A_{\text {iso }}\left({ }^{\prime} \mathrm{H}\right)$. For $\mathrm{H}_{2} \mathrm{CN}$ the direct contributions are given in Table 3; the indirect contributions are obtained from the difference between the direct contributions and the total result. As found for the heavier centers the indirect contribution to $A_{\text {iso }}\left({ }^{\prime} \mathrm{H}\right)$ is much smaller in the DFT calculations (16-21 G) than in the MRD-CI $/ B_{K}$ treatment $(\approx 40 \mathrm{G})$. However, this is compensated for by larger direct contributions so that in total very similar results are obtained in both methods. The deviations between the various functionals arise due to differences within the indirect contribution while the direct contributions are identical.

The reason for the higher direct contribution can be seen from Fig. I which compares the shape of the SOMO obtained from a DFT-BLYP calculation (Fig. la) to the form of the SOMO obtained from an ROHF calculation (Fig. 1b). In the DFT treatment the density of the SOMO is more delocalized from the nitrogen center to the hydrogen centers. The natural orbitals which were employed with the MRD$\mathrm{CI} / \mathrm{B}_{\mathrm{K}}$ treatment are somewhat more compact than the SOMO of the ROHF calculation as can be seen from the direct contribution given for the MRD-Cl/ $\mathrm{B}_{\mathrm{K}}$ calculation.

Two further examples known as problematic cases shall conclude the present work. Table 4 contains the results obtained for $\mathrm{O}_{2}$ and NO. In both molecules the isotropic hfccs are solely determined by spin polarization effects. As found for the heavier centers of $\mathrm{H}_{2} \mathrm{CN}$ and $\mathrm{H}_{2} \mathrm{CO}^{+}$, the absolute values of the calculated isotropic hfccs obtained by the various DFT functionals are too small ( $30 \%-60 \%)$.

The examples given in the present investigation underscore that at least the functionals used in this work (and the PW86 functional used by Eriksson et al. [13]) are not accurate enough to describe the interaction between the singly occupied and the doubly occupied shells sufficiently well. Comparing to ab initio data the contribution to isotropic hfcc arising due to these spin polarization effects are computed absolutely too low. Let us first consider those cases where the direct contribution vanishes due to symmetry reasons. As already discussed the net spin density at the center under consideration solely arises from the interaction between the singly and doubly occupied shells so that the indirect contributions represent an observable. The non-local nature of this interaction is obvious since the density of the unpaired electron vanishes at the point where the effect of the interaction is measured, i.e. at the position of the center under consideration. The difficulties in describing nonlocal effects within DFT are known [35] and explain the errors in the isotropic hfcc computed with the DFT method.

If direct contributions are important a cancellation takes place leading, for example, to accurate iso- 

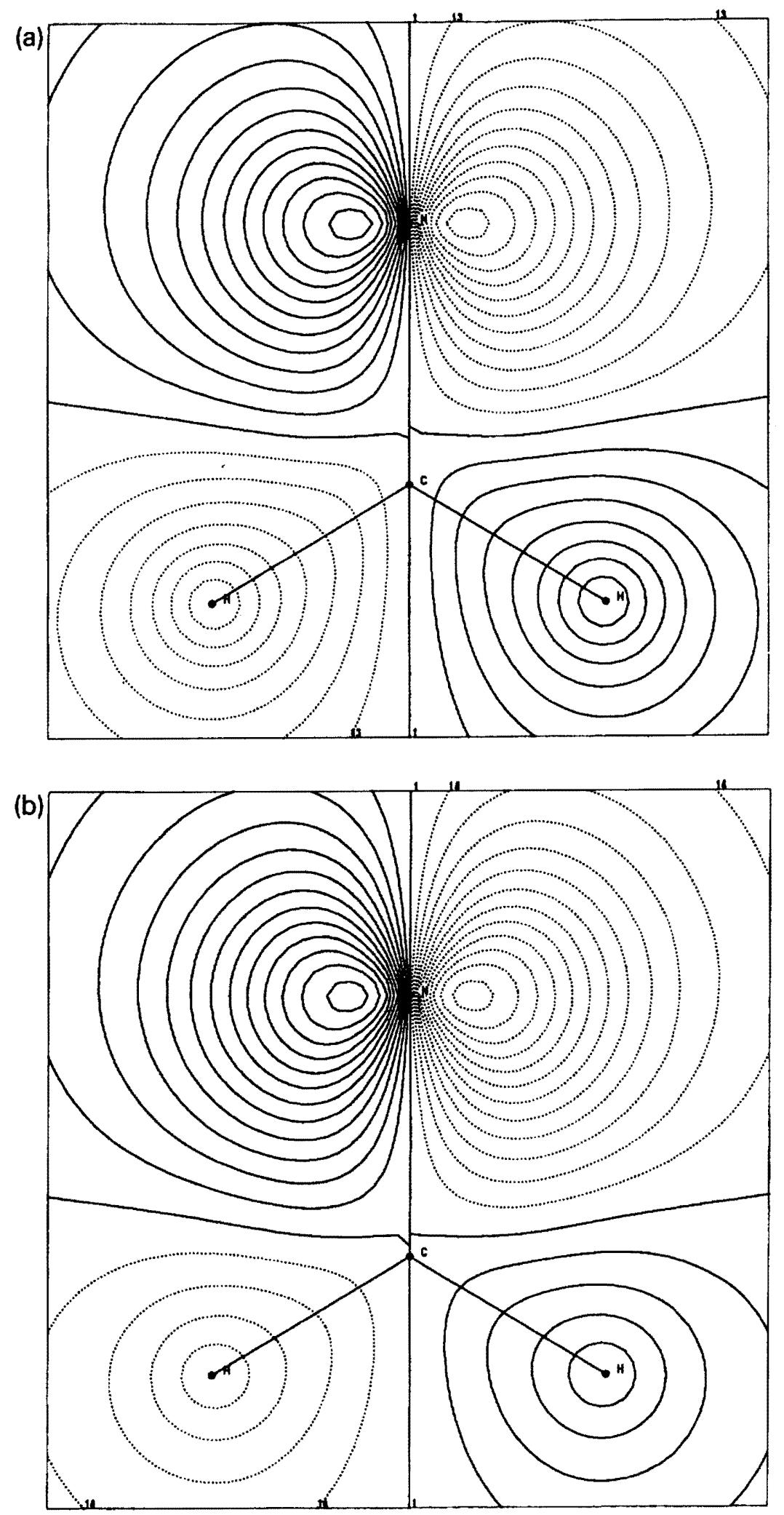

Fig. 1. Shape of the singly occupied orbital (SOMO) (a) obtained from the BLYP-DFT calculation, (b) obtained from an ROHF calculation. 
tropic hfccs of $\beta$ protons as shown for $\mathrm{H}_{2} \mathrm{CN}$ and $\mathrm{H}_{2} \mathrm{CO}^{+}$. In such cases neither the direct nor indirect contributions represent observables. Therefore it is unclear whether this represents an error cancellation or arises from the differences in the description of correlation effects in the MRD-CI/ $\mathrm{B}_{\mathrm{K}}$ treatment and the DFT method.

Comparing the various examples it is obvious that the error made by DFT depends on the system under consideration. While the PW86 functional performs perfectly for $A_{\text {iso }}\left({ }^{17} \mathrm{O}\right)$ in the $\mathrm{OH}$ molecule it gives bad results in the case of $\mathrm{H}_{2} \mathrm{CO}^{+}$, although in both molecules the unpaired electron is mainly located at the oxygen. Further studies seem to be necessary to understand the underlying reasons for this behaviour. Such investigations are also interesting because isotropic hyperfine coupling constants directly probe the electron spin density at the nuclei and therefore provide a valuable measure for the quality of approximate spin density functionals.

\section{Acknowledgement}

The authors thank Professor S.D. Peyerimhoff and Professor F. Grein for their interest and for fruitful discussions during the progress of this work. Part of this work was financially supported by the Deutsche Forschungsgemeinschaft (DFG) in the framework of project EN 197/2-3 and the Leibniz prize awarded to Professor S.D. Peyerimhoff. Computer time was provided by the computing center of the RWTH Aachen and the Centro Svizzero di Calcolo Scientifico in Manno.

\section{References}

[1] D. Feller and E.R. Davidson, J. Chem. Phys. 80 (1984) 1006.

[2] D. Feller and E.R. Davidson, in: Theoretical models of chemical bonding, ed. Z.B. Maksic (Springer, Berlin, 1991).

[3] D.M. Chipman, J. Chem. Phys. 91 (1989) 5455.

[4] I. Carmichael, J. Phys. Chem. 95 (1991) 108.

[5] B. Engels, J. Chem. Phys. 100 (1994) 1380.

[6] H.U. Suter and B. Engels, J. Chem. Phys. 100 (1994) 2936.
[7] A.D. Becke, J. Chem. Phys. 88 (1988) 2547.

[8] J.P. Perdew, Phys. Rev. B 33 (1986) 8822.

[9] J.P. Perdew and Y. Wang, Phys. Rev. B 33 (1986) 8822.

[10] J.P. Perdew, in: Electronic structures of solids, eds. P. Zieschle and $\mathrm{H}$. Eschrig (Akademie, Berlin, 1991).

[11] C. Lee, W. Yang and R.G. Parr, Phys. Rev. B 37 (1988) 785.

[12] L.A. Eriksson, J. Wang, R.J. Boyd and S. Lunell, J. Phys. Chem. 98 (1994) 792.

[13] L.A. Eriksson, O.L. Malkina, V.G. Malkin and D.R. Salahub J. Chem. Phys. 100 (1994) 5066, and references therein.

[14] N. Ishii and T. Shimizu, Phys. Rev. A 48 (1994) 1691.

[15] J. Kong, L.A. Eriksson and R.J. Boyd, Chem. Phys. Letters 217 (1994) 24.

[16] L. Fan and T. Ziegler, J. Chem. Phys. 94 (1991) 6057.

[17] M.J. Frisch, G.W. Trucks, H.B. Schlegel, P.M.W. Gill, B.G. Johnson, M.W. Wong, J.B. Foresman, M.A. Robb, M. HeadGordon, E.S. Replogle, R. Gomperts, J.L. Andres, K. Raghavachari, J.S. Binkley, C. Gonzalez, R.L. Martin, D.J. Fox, D.J. DeFrees, J. Baker, J.J.P. Stewart and J.A. Pople, GAUSSIAN 92/DFT, Revision G.l (Gaussian, Inc., Pittsburgh PA, 1993).

[18] S.H. Vosko, L. Wilk and M. Nusair, Can. J. Phys. 58 (1980) 1200.

[19] D.M. Ceperly and B.J. Alder, Phys. Rev. Letters 45 (1980) 566.

[20] F.B. Duijneveldt, Techn. Rept. RJ945, IBM Research Lab, San Jose, CA (1971).

[21] W. Kutzelnigg, U. Fleischer and $M$. Schindler, in: NMR basic principles and progress, Vol. 23 (Springer, Berlin, 1990) p. 165.

[22] S. Huzinaga and Y. Sakai, J. Chem. Phys. 50 (1969) 1371.

[23] W. Kohn and L.J. Sham, Phys. Rev. 140 (1965) Al11 3.

[24] T.C. Steimle, D.R. Woodward and J.M. Brown, J. Chem. Phys. 85 (1986) 1276.

[25] B. Engels and S.D. Peyerimhoff, Mol. Phys. 67 (1989) 583.

[26] W. Ubachs, J.J. ter Meulen and A. Dymanus, Can. J. Phys. 62 (1984) 1374.

[27] J.A. Coxon, K.V.L.N. Santry, J.A. Austin and D.H. Levy, Can. J. Phys. 57 (1979) 619.

[28] L.B. Knight and J. Steadman, J. Chem. Phys. 80 (1984) 1018.

[29] S. Yamamoto and S. Saito, J. Chem. Phys. 96 (1992) 4157.

[30] P. Bündgen, F. Grein and B. Engels, submitted for publication.

[31] P. Kristiansen, J. Mol. Spectry. 66 (1977) 177.

[32] W.L. Meerts and A. Dynamus, J. Mol. Spectry. 44 (1972) 320.

[33] G. Gazzoli, C.D. Espositi, P.G. Favero, G. Severi, Nuovo Cimento B 62 (1981) 243.

[34] D.M. Chipman, I. Carmichael and D. Feller, J. Phys. Chem. 95 (1991) 4702

[35] J.P. Perdew, Intern. J. Quantum Chem. Quantum Chem. Symp. 27 (1993) 93. 